\title{
miR-34a inhibits the metastasis of osteosarcoma cells by repressing the expression of $\mathrm{CD44}$
}

\author{
HAIEN ZHAO ${ }^{1,2}$, BAOAN MA $^{1}$, YUCAI WANG ${ }^{1}$, TAO HAN $^{1}$, LIANHE ZHENG $^{1}$, CONG SUN $^{1}$, \\ TAO LIU ${ }^{1}$, YINGLONG ZHANG ${ }^{1}$, XIUCHUN QIU ${ }^{1}$ and QINGYU FAN ${ }^{1}$ \\ ${ }^{1}$ Department of Orthopaedic Surgery, Tangdu Hospital, The Fourth Military Medical University, Xi'an, \\ Shaanxi 710038; ${ }^{2}$ Xi'an Research Institute of Hi-Tech., Hongqing, Xi'an 710025, P.R. China
}

Received November 1, 2012; Accepted December 3, 2012

DOI: $10.3892 / o r .2013 .2234$

\begin{abstract}
Osteosarcoma is the most common type of malignant bone tumor in children and adolescents and approximately $30 \%$ of patients develop lung metastasis, which is the leading cause of mortality. In this study, we investigated the role of miR-34a in the invasion and metastasis of osteosarcoma cells by examining its expression level and functional pattern in these cells. miR-34a mimics were transfected into the highly metastatic subline, F5M2, and into the F4 subline with low metastatic potential of the paired human osteosarcoma cell line, SOSP-9607. Cell viability patterns, cell migration and alterations in gene expression levels were assessed by realtime PCR, and changes in protein levels were assessed by immunocytochemistry and western blot analysis. The ectopic overexpression of miR-34a significantly inhibited the migration and invasive ability of osteosarcoma cells by repressing the expression of CD44. These data suggest that miR-34a plays a tumor suppressor role in the metastasis of osteosarcoma cells by repressing the expression of CD44. Of note, studies have also suggested that the CD44 protein correlates with the metastatic potential of several malignant tumors. Therefore, it can be concluded that through the inhibition of CD44 expression levels, miR-34a plays a significant role in the migration and invasion of osteosarcoma cells.
\end{abstract}

\section{Introduction}

Osteosarcoma is the most common type of malignant bone tumor in children and adolescents. Clinical studies have reported that this tumor has a poor prognosis, even with current treatment options, such as surgical intervention and chemotherapy $(1,2)$. Over the last few decades, neoadjuvant chemotherapy and

Correspondence to: Professor Qingyu Fan, Department of Orthopaedic Surgery, Tangdu Hospital, The Fourth Military Medical University, 1 Xinyi Road, Xi'an, Shaanxi 710038, P.R. China E-mail: 53383365@qq.com

Key words: osteosarcoma, miR-34a, CD44, cancer stem cell, migration, invasion surgery have been considered as successful treatments for osteosarcoma and the limb salvage rate has significantly increased, considerably raising the survival to $65-75 \%$. However, approximately $30 \%$ of osteosarcoma patients develop metastasis, particularly pulmonary metastasis, which is the leading cause of mortality (3). Thus, it is crucial to identify metastasisassociated molecules and to elucidate the mechanisms involved in pulmonary metastasis from osteosarcoma. A number of studies have shown that specific microRNAs (miRNAs) are aberrantly expressed in malignant hepatocellular carcinoma (HCC) cells or tissues compared to non-malignant hepatocytes or tissues $(4,5)$. miRNAs are metastasis-associated molecules during the progression and metastasis of osteosarcoma.

miRNAs are a class of small non-coding regulatory RNA molecules (21-23 nucleotides) encoded in the genome, with profound impacts on various cellular processes $(6,7)$. These small molecules mainly bind imperfectly by base pairing to the 3' untranslated region (3'UTR) of target mRNAs and repress protein expression through either degradation or silencing (7-9). Evidence has shown that miRNA mutations or misexpression correlate with various human cancers, where they were thought to function as tumor suppressors or oncogenes by regulating the expression of other tumor suppressors or oncogenes (8-10). However, the mechanisms by which miRNAs mediate tumor metastasis have only been recently investigated and have not been fully elucidated.

miR-34 family members, consisting of miR-34a, b and c, are direct transcription targets of the tumor suppressor protein p53 (11). miR-34a has been reported to induce cell apoptosis, cell cycle arrest or cell senescence depending on the cell type analyzed (11-13). Some targets of miR-34a have been recently identified including cyclin-dependent kinase (CDK)4/6, cyclin D1 (CCND1), cyclin E2 (CCNE2), E2F transcription factor 3 (E2F3), Bcl-2, MYCN, c-Met, sirtuin 1 (SIRT1) (14-17). However, its role in mediating osteosarcoma metastasis to the lungs has only been recently investigated and remains largely ambiguous.

The current study was carried out to investigate the potential role of miR-34a in the invasion and metastasis of osteosarcoma cells. We investigated the expression level and functional pattern of miR-34a in osteosarcoma cells. This was performed by the quantification of miR-34a in the highly metastatic subline, F5M2, and in the F4 subline with low 
metastatic potential of the paired human osteosarcoma cell line, SOSP-9607. The transfection outcomes were subsequently assessed according to cell viability patterns, cell migration and alterations in gene expression by real-time PCR, as well as the protein level by immunocytochemistry (ICC) and western blot analysis. Functional analysis and sequence analysis were then carried out by the transfection of miR-34a mimics or inhibitors into the highly metastatic subline, F5M2, with a low endogenous miR-34a level.

In the present study, we demonstrated that the miR-34a expression level in the F4 human osteosarcoma cells with a low metastatic potential was higher than that in the highly metastatic F5M2 cells. Subsequently, we investigated the effects of the ectopic expression of miR-34a on F5M2 cells, and showed that miR-34a inhibited the invasion and metastasis of osteosarcoma cells in vitro. Sequence analysis suggested an interaction between the 3'UTR of CD44 mRNA and miR-34a. Therefore, we hypothesized that miR-34a can repress the expression of CD44 and analyzed its functions in human osteosarcoma cells.

\section{Materials and methods}

Cell culture. A pair of human osteosarcoma cell lines with different pulmonary metastatic potentials, the highly metastatic subline, F5M2, and the F4 subline with low metastatic potential, originating from the human osteosarcoma cell line, SOSP-9607, were established by Dr X. Chen in our laboratory (18). The F5M2 and F4 cell sublines were cultured in complete RPMI-1640 medium (HyClone, Logan, UT, USA) supplemented with $10 \%$ fetal bovine serum (Sijiqing Co. Hangzhou, China) in $5 \% \mathrm{CO}_{2}$ at $37^{\circ} \mathrm{C}$.

Transfection. In this study, transfection was performed with Lipofectamine 2000 Reagent (Invitrogen, Carlsbad, CA, USA) according to the manufacturer's instructions. miR-34a mimics, mimic negative controls, miR-34a inhibitor and negative controls were purchased from RiboBio (Guangzhou, China). A low concentration of $20 \mathrm{nM}$ or a high concentration of $50 \mathrm{nM}$ of the mimics were used for each transfection in the migration, invasion, apoptosis and proliferation assays, compared with the F5M2 cells transfected with the mimic negative controls, miR-34a inhibitor and negative controls. The efficiency of the miR-34a transfection was measured by quantitative real-time PCR.

Quantitative real-time PCR analysis. miR-34a, CD44 mRNA expression was measured by real-time PCR. Total RNA was extracted using TRIzol reagent (Invitrogen) according to the manufacturer's instructions. For miR-34a quantitative real-time PCR, total RNA was reverse transcribed with a miR-sepicfic primer (RiboBio) and then quantitative real-time PCR was performed with a miR-sepicfic primer using the ABI PRISM 7500 real-time PCR system (Applied Biosystems, Bedford, MA, USA). U6 was used as the normalization control. Quantitative real-time PCR for CD44 mRNA was performed with CD44 mRNA primers (forward, 5'-CATCTACCCCA GCAACCCTA-3'; and reverse, 5'-ACTGTCTTCGTCTGGG ATGG-3') and the relative expression level normalized to GAPDH was calculated using the comparative $\mathrm{Ct}$ method.
Transwell assay. For migration assay, we used the $6.5-\mathrm{mm}$ transwell insert (pore size, $8 \mu \mathrm{m}$; 24-well insert; Corning) to examine the effect of miR-34a on the migration and invasion of the F5M2 and F4 cells. Cells were serum-free-starved overnight, and then harvested and resuspended in migration medium (RPMI-1640 medium free with fetal bovine serum). Cell suspension (5,000 cells) in $100 \mu 1$ migration medium was then added to the inside of the insert. RPMI-1640 medium (600 $\mu \mathrm{l}$ ) containing $20 \%$ fetal bovine serum was added to the lower chambers outside of the insert. After the cells were incubated for $48 \mathrm{~h}$, the non-invading cells that remained on the upper surface of the insert were removed with a cotton swab. The invading cells on the lower surface of the insert were fixed with $95 \%$ ethanol for $30 \mathrm{~min}$ and then stained with $0.1 \%$ crystal violet for $5 \mathrm{~min}$. The number of cells on the lower surface was counted under a microscope in 6 randomized visual fields of each insert at a magnification of $\mathrm{x} 100$.

Invasion assay was carried out in a similar manner as the migration assay but the inserts were pre-coated with $40 \mu \mathrm{l}$ BD Matrigel (1:3 dilution; BD Biosciences, San Jose, CA USA) according to the manufacturer's instructions.

Wound healing assay. Adhered cell monolayers on 6-well plates were scratched with a $20-\mu 1$ pipette tip (Eppendorf) and grown in RPMI-1640 medium with 10\% fetal bovine serum (Sijiqing Biological Engineering Materials Co., Ltd., Hangzhou, China) with $5 \% \mathrm{CO}_{2}$ at $37^{\circ} \mathrm{C}$. Wound healing capacity was monitored under a microscope after $0,12,24$ and $36 \mathrm{~h}$.

Apoptosis and proliferation assay. For the apoptosis assay, the cells were stained with FITC-conjugated anti-Annexin V antibody. The Annexin V-FITC apoptosis detection kit (BD Pharmingen, San Diego, CA, USA) was employed to analyze cell apoptosis with flow cytometry (BD Biosciences FACSAria cell sorter).

For the proliferation assay, the proliferative capacity of the cells was evaluated with an MTT assay, which was performed following standard procedure in 96-well plates. Cells were seeded at a density of 2,000 cells/well containing $100 \mu \mathrm{l}$ of culture medium and cultured overnight. At 24-h intervals, $20 \mu \mathrm{l}$ of $5 \mathrm{mg} / \mathrm{ml}$ MTT (dimethyl thiazolyl diphenyl tetrazolium; Sigma) were added to each well and then the cells were incubated for $4 \mathrm{~h}$ at $37^{\circ} \mathrm{C}$. The medium was then removed, and $100 \mu \mathrm{l}$ of dimethyl sulfoxide (DMSO) were added to each well to dissolve the formazan. The optical density (OD) was evaluated by measuring the absorbance.

Western blot analysis and ICC. The CD44 expression level was analyzed by western blot analysis using CD44 mouse monoclonal antibody (Abcam) and anti- $\beta$-actin mouse monoclonal antibody (Epitomics, Inc., Burlingame, CA, USA). ICC was performed with CD44 mouse monoclonal antibody (Abcam) and the Envision ${ }^{\mathrm{TM}}$ Detection kit (Gene-Tech Co., Ltd., Shanghai, China) according to a standard method.

Luciferase reporter assay. F5M2 cells were transfected in 6-well plates with $2 \mu \mathrm{g}$ of CD44 3'UTR (containing the binding site of miR-34a) luciferase reporter plasmid per well, using Lipofectamine 2000 (Invitrogen). Cells in each well were also co-transfected with 100 pmol of each miR-34 mimics, nega- 
tive control mimics, or miR-34a inhibitors as indicated. Cells were collected $48 \mathrm{~h}$ after transfection, and luciferase assays were performed using a dual-luciferase reporter assay system (Promega). Luciferase activity was normalized relative to the control activity as previously described (19).

Statistical analysis. All statistical analyses were performed using SPSS 17.0 software. The experiments were repeated 3 times independently and the results are presented as the means \pm SD. The differences between groups were analyzed using Student's t-tests; $\mathrm{P}<0.05$ was considered to indicate a statistically significant difference.

\section{Results}

miR-34a expression levels are lower in F5M2 cells than in F4 cells. In this study, the F5M2 and F4 cell sublines were chosen as the objectives since they originate from the same maternal cell line, SOSP-9607 (human osteosarcoma cell line), but display dramatically significant differences in metastatic and invasive potential (18).

Of all potential miRNAs, we focused on miR-34a since it is one of the most obviously altered miRNAs and is underexpressed in mouse hepatoma cells with a high lymphatic metastatic potential and in highly metastatic human prostate cancer cells $(20,21)$. However, the functional mechanism of miR-34a in these cancers remains unclear.

In order to investigate the differential expression of miR-34a in the osteosarcoma cell sublines with different metastatic potential, we compared the miR-34a expression between the F5M2 and F4 cell sublines by quantitative real-time PCR. Consistent with the results obtained for the mouse hepatoma cells and for the highly metastatic human prostate cancer cells, real-time PCR revealed that the expression level of miR-34a in the highly metastatic F5M2 cells was lower than that in the F4 cells with low metastatic potential. This difference in expression level was statistically significant $(\mathrm{P}<0.05$; Fig 1$)$. These results suggest that the downregulation of miR-34a is involved in the metastatic and invasive potential of human osteosarcoma cells.

F5M2 cells significantly overexpress miR-34a after transfection. F5M2 cells were transfected with the miR-34a mimics at 2 different concentrations, 20 or $50 \mathrm{nM}$. The control groups included F5M2 cells that were untreated or transfected with the mimic negative controls, miR-34a inhibitor and negative controls. To verify the efficiency of the transfection, the expression of miR-34a was measured by quantitative real-time PCR of total RNA $48 \mathrm{~h}$ after transfection.

The results from real-time PCR showed that the expression of miR-34a significantly increased in the F5M2 cells after transfection with the miR-34a mimics, compared with the control groups $(\mathrm{P}<0.05$; Fig. 1). The results also showed that miR-34a expression in the F5M2 cells transfected with a high concentration of mimics $(50 \mathrm{nM})$ was significantly higher than that in the cells transfected with a low concentration of mimics $(20 \mathrm{nM})$. Thus, the transfection was successful. The higher the concentration of the miR-34a mimics, the higher the expression of the miR-34a in the F5M2 cells after transfection.

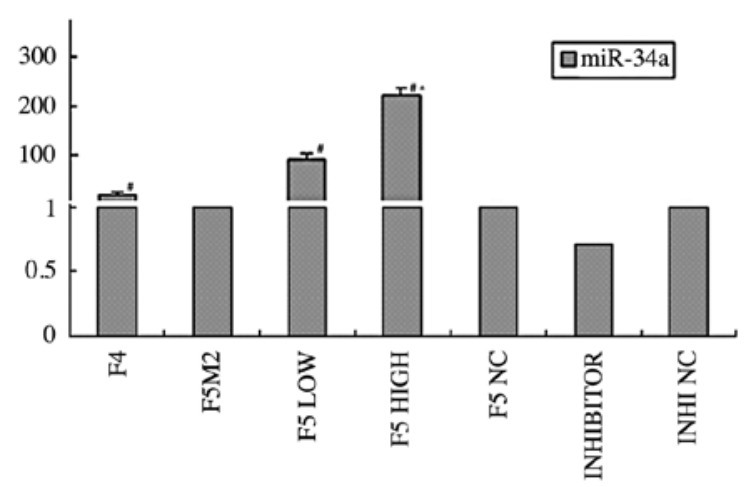

Figure 1. RT-PCR. Total RNA was extracted from the cells, and the level of miR-34a was analyzed by quantitative real-time PCR. The results showed that miR-34a expression in F5M2 was significantly lower than that in F4 cells $\left({ }^{\#} \mathrm{P}<0.05\right)$. The miR-34a level increased markedly in F5M2 cells after transfection with miR-34a mimics ( $\left.{ }^{*} \mathrm{P}<0.05\right)$, compared with F5M2 cells untreated or transfected with mimic negative controls (NC) or inhibitors. There was no significant difference between the F5M2 cells untreated and transfected with mimic NC or inhibitors. miR-34a expression in F5M2 cells transfected with a high concentration of mimics $(50 \mathrm{nM})$ was significantly higher than that in cells transfected with a low concentration of mimics $(20 \mathrm{nM})\left({ }^{*} \mathrm{P}<0.05\right)$. The results represent the relative level of miR-34a in F5M2 cells.

$C D 44 m R N A$ is a target of $m i R-34 a$. The prediction of miR-34a putative target genes was performed using bioinformatics algorithms based on sequence similarity between miRNAs and mRNAs. We used TargetScan (http://www.targetscan.org/) (22) and PicTa'r (http://pictar.mdc-berlin.de/) $(23,24)$ to predict the targets of miR-34a. Remarkably, the programs predicted that CD44 was one of the targets of miR-34a. Liu et al established miR-34a as a critical negative regulator of CD44+ $\mathrm{PCa}$ cells and CD44 itself as an important target of miR-34a (21). Therefore, we hypothesized that miR-34a may alter F5M2 cell migration and invasion by regulating the expression of CD44. To verify this hypothesis, we examined the expression level of CD44 in F5M2 cells after transfection with miR-34a mimics by ICC and western blot analysis.

ICC analysis showed that the staining intensity of CD44 in the F5M2 cells was stronger than that in the F4 cells; this was significantly reduced in the F5M2 cells after transfection with miR-34a mimics (Fig. 2A). The results from western blot analysis were similar to those from ICC analysis, in that the expression level of CD44 in the F5M2 cells was significantly higher than that in the F4 cells and that the expression of CD44 in the F5M2 cells markedly decreased after transfection with miR-34a mimics, compared with the F5M2 cells untreated or treated with the negative controls (Fig. 2B). Both in western blot analysis and ICC, the expression of CD44 had the same tendency; the F5M2 cells treated with $50 \mathrm{nM}$ miR-34a mimics expressed lower levels of CD44 than the cells treated with $20 \mathrm{nM}$ miR-34a mimics $(\mathrm{P}<0.05)$. There was a negative correlation between the CD44 levels and the exogenous miR-34a levels. The overexpression of miR-34a may thus inhibit the expression of CD44. Therefore, CD44 mRNA is a possible target of miR-34a.

To further prove that the changes in the protein levels were a direct effect of miR-34a, we fused the 3'UTRs of CD44 to a luciferase reporter construct. The results demonstrated that the luciferase activity of the miR-CD44 UTR construct 

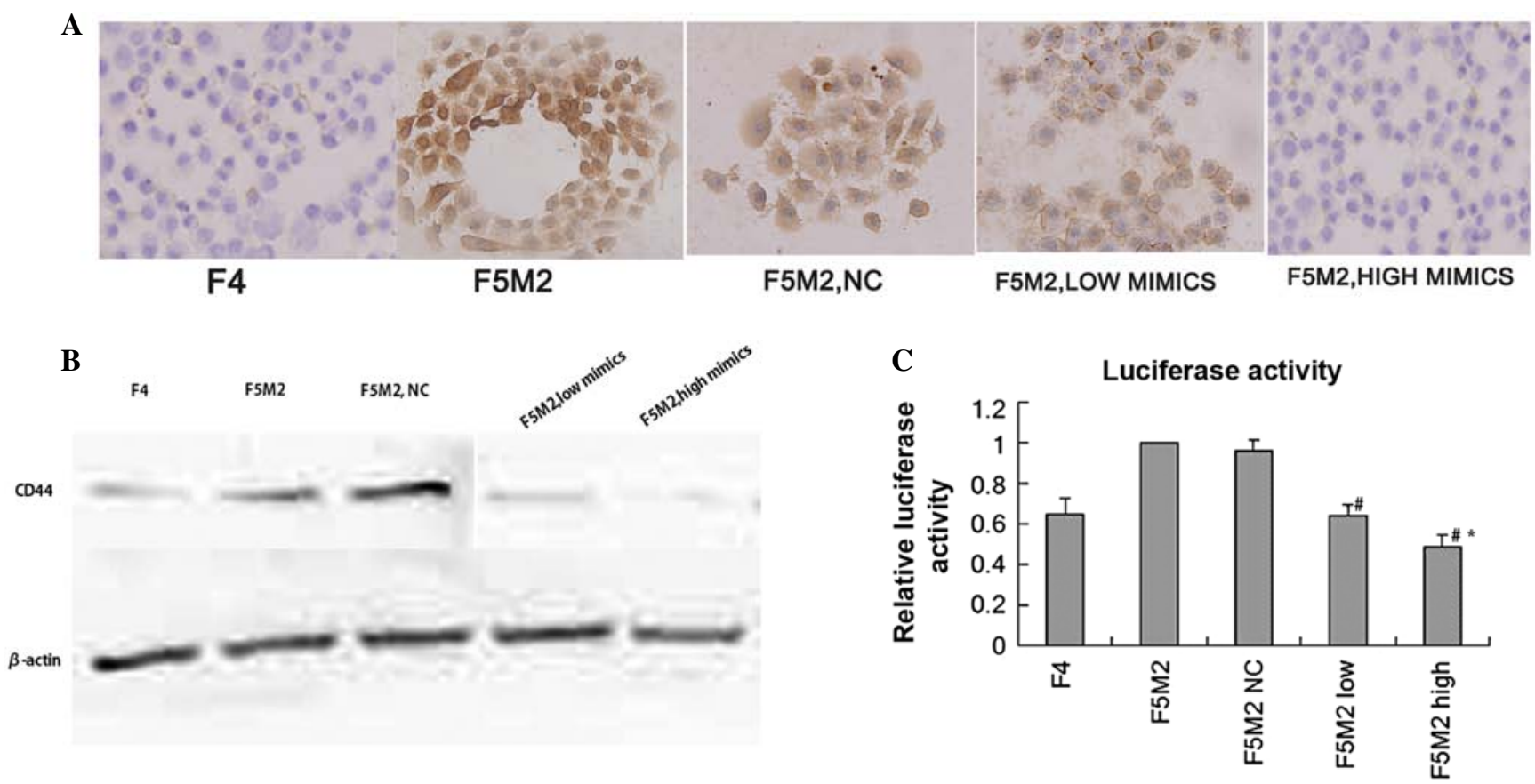

Figure 2. (A) Immunocytochemistry (ICC) showed that CD44 expression in F5M2 cells was significantly higher than in F4 cells. After transfection with miR-34a mimics, CD44 expression in F5M2 cells was lower than in F5M2 cells untreated or transfected with mimic negative controls (NC) or inhibitors. Of note, CD44 expression in F5M2 cells transfected with a high dose of miR-34a (50 nM) was lower than in cells treated with a low dose of miR-34a (20 nM). CD44 was expressed mainly in the membrane of the osteosarcoma cells. (B) Consistent with the results of ICC, western blot analysis showed that CD44 expression was significantly higher in F5M2 cells than in F4 cells. After transfection with a low dose of miR-34a, CD44 expression in F5M2 cells was lower than in F5M2 cells untreated or transfected with mimic NC or inhibitors. After transfection with a high dose of miR-34a mimics, CD44 expression was almost abolished. CD44 expression in F5M2 cells transfected with a high dose of miR-34a $(50 \mathrm{nM})$ was lower than that in cells treated with a low dose of miR-34a $(20 \mathrm{nM}) . \beta$-actin was used as the internal reference positive control. (C) The results of luciferase assay demonstrated that the luciferase activity of the miRCD44 UTR construct was significantly inhibited after the introduction of the miR-34a mimics, compared with the untreated F5M2 cells ( ${ }^{*} \mathrm{P}<0.05$ ). Luciferase activity in F5M2 cells transfected with a high dose of miR-34a (50 nM) was lower than that in cells treated with a low dose of miR-34a (20 nM) ("P<0.05).

was significantly inhibited after the introduction of miR-34a mimics (Fig. 2C; $\mathrm{P}<0.05$ ). Consistent with the results reported previously by others $(21,25)$, the transfection of miR-34a mimics effectively modulated the luciferase reporter gene expression compared with the control groups. The results of luciferase reporter assay demonstrated that CD44 was a direct target of miR-34a in the F5M2 cells.

miR-34a significantly decreases the migration and invasive ability of F5M2 cells. Consistent with the conclusions of the metastatic potential of F5M2 cells presented in the study by Chen et al (18), in our study, F5M2 cells displayed a significantly higher migration and invasive capability than F4 cells in vitro, as shown by transwell assay (Fig. 3A and B).

To explore the potential role of miR-34a in osteosarcoma cell metastasis, we examined the cell motility and invasive capability after the ectopic expression of miR-34a in F5M2 cells, which had been verified to underexpress endogenous miR-34a previously.

Transwell assay without Matrigel was employed to examine the migration capability. Cells that penetrated the membrane and reached the under side of the transwell were recorded after incubation for $48 \mathrm{~h}$. The results showed that the ectopic overexpression of miR-34a significantly repressed the chemotaxis of F5M2 cells, compared with the F5M2 cells that were untransfected or transfected with the mimic controls (Fig. 3A and $\mathrm{C} ; \mathrm{P}<0.05)$.
Transwell inserts with a layer of Matrigel on top of the inserts were employed to examine the invasive capability. Cells that penetrated both Matrigel and membrane were counted after incubation for $48 \mathrm{~h}$. The results showed that ectopic miR-34a expression in the F5M2 cells significantly inhibited their invasive ability (Fig. 3B and D; $\mathrm{P}<0.05$ ), which was consistent with the results obtained for migration.

Additionally, the cell motility of F5M2 cells transfected with a high concentration of mimics was significantly weaker than that of F5M2 cells transfected with a low concentration of mimics, as shown by both the migration and invasion assay. Taken together, these results demonstrate that miR-34a inhibits the F5M2 cell migration and invasion potential in vitro.

miR-34a significantly decreases wound healing capacity of F5M2 cells. To compare the polarized migration of F5M2 and F4 cells, we employed the scratch wound cell model. The results showed that in the F5M2 cells, the scratch wounds healed faster than those in the F4 cells (Fig. 4; $\mathrm{P}<0.05$ ). This model also showed that ectogenic miR-34a significantly decreased the wound healing capacity of F5M2 cells, compared with those cells untreated or transfected with the negative controls (Fig. 4 and Table I). These results demonstrated that the inhibitory effect of miR-34a was dependent on the concentration of miR-34a mimics (data not shown). The higher the concentration of the miR-34a mimics, the slower the rate at which the F5M2 cells closed the scratch wounds after transfection. 

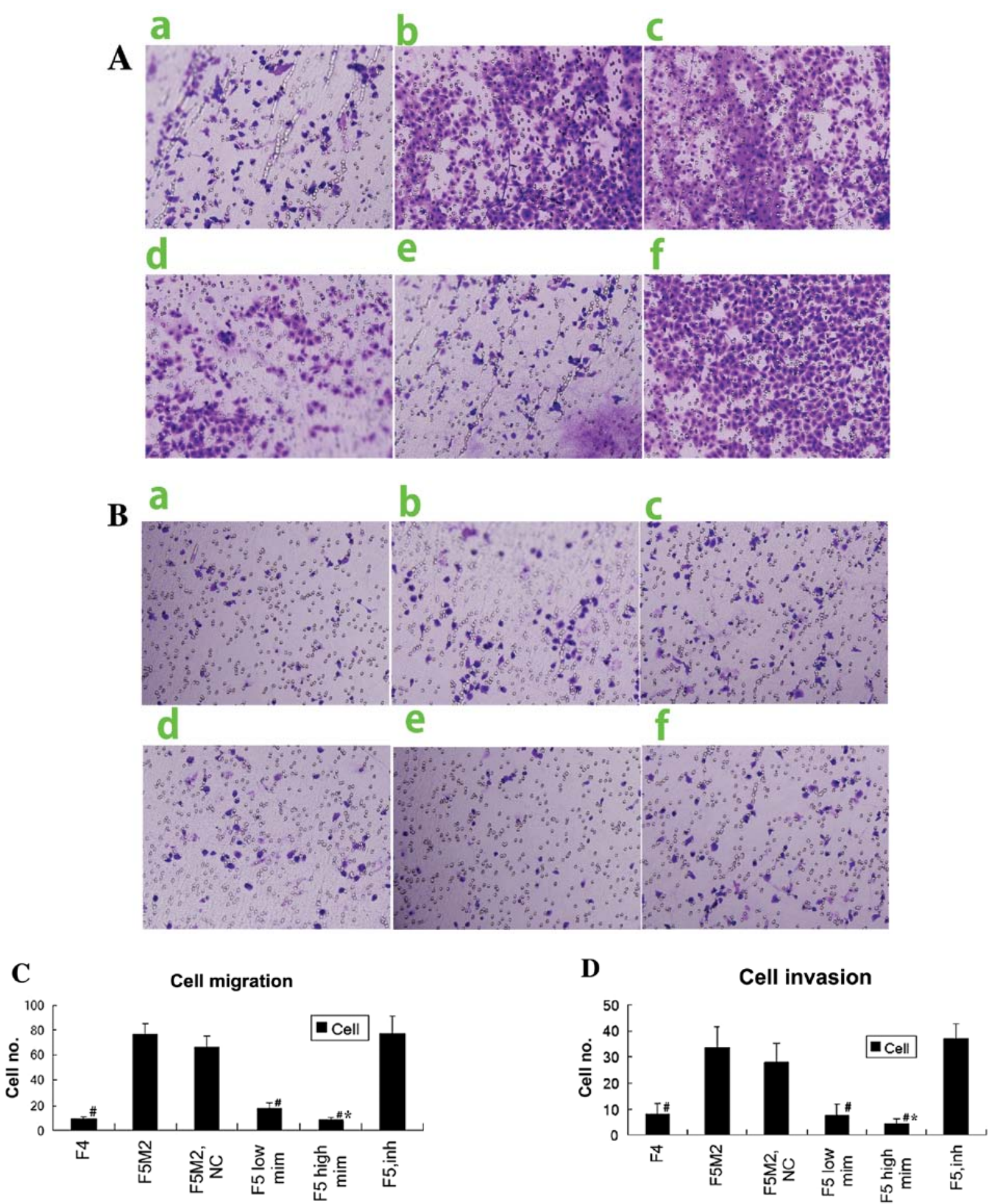

Figure 3. Transwell assay showed that miR-34a negatively regulated F5M2 cell migration and invasion in vitro. (A and C) The cell migration number of F4 or F5M2 cells transfected with miR-34a mimics was significantly lower than that of F5M2 cells untreated or treated with mimic negative controls (NC) or inhibitors. The differences between F5M2 cells transfected with miR-34a mimics and untreated F5M2 cells was significant ( $\left.{ }^{\#} \mathrm{P}<0.05\right)$. There was no significant difference between F5M2 cells untreated and transfected with mimic NC or inhibitors. (B and D) The invasive cell number of F4 or F5M2 cells transfected with miR-34a mimics was significantly lower than that of F5M2 cells untreated or treated with NC. There was no significant difference between F5M2 cells untreated and transfected with mimic NC or inhibitors. The differences between F5M2 cells transfected with miR-34a mimics and F5M2 cells untreated were significant $\left({ }^{*} \mathrm{P}<0.05\right) .{ }^{\#} \mathrm{P}<0.05$, compared with F5M2. ${ }^{*} \mathrm{P}<0.05$, comparison between high miR-34a and low miR-34a. (A and B) (a) F4; (b) F5M2; (c) F5M2 with NC; (d) F5M2 with low miR-34a; (e) F5M2 with high miR-34a; (f) F5M2 with miR-34a inhinibitor. inh, inhibitor.

miR-34a influences apoptosis and proliferation of F5M2 cells. The results of flow cytometry showed that there was no statistically significant difference either between F5M2 and F4 cells, or between F5M2 cells untreated or transfected with miR-34a mimics and negative controls (Fig. 5A and B). Therefore, miR-34a had little effect on cell apoptosis.

In order to investigate the effects of miR-34a on cell proliferation, MTT assay was performed. The proliferation 
Table I. Wound closure rate of F4 or F5M2 transfected with miR-34a mimics, untreated or treated with the negative controls.

\begin{tabular}{llccc}
\hline & \multicolumn{4}{c}{ Time (h) } \\
\cline { 2 - 5 } Cell transfection & 0 & 12 & 24 & 36 \\
\hline F4 & 0 & $0.42 \pm 0.08$ & $0.62 \pm 0.02^{\mathrm{a}}$ & $0.90 \pm 0.13^{\mathrm{a}}$ \\
F5M2 & 0 & $0.41 \pm 0.12$ & $0.83 \pm 0.16$ & $0.99 \pm 0.08$ \\
F5M2, NC & 0 & $0.45 \pm 0.12$ & $0.85 \pm 0.06$ & $0.06 \pm 0.03$ \\
F5M2, miR-34a & 0 & $0.43 \pm 0.07$ & $0.61 \pm 0.08^{\mathrm{a}}$ & \\
\hline
\end{tabular}

The would closure rate of F4 or F5M2 cells transfected with miR-34a mimics was significantly lower compared to cells untreated or treated with the negative controls $(\mathrm{P}<0.05)$.There was no significant difference between F5M2 cells untreated or treated with the negative controls (NC) $(\mathrm{P}>0.05)$. Wound closure rate was defined as closed width/h (means $\pm \mathrm{SD}$ ). ${ }^{\mathrm{a}} \mathrm{P}<0.05$, compared with F5M2 cells untreated at the same time-point.

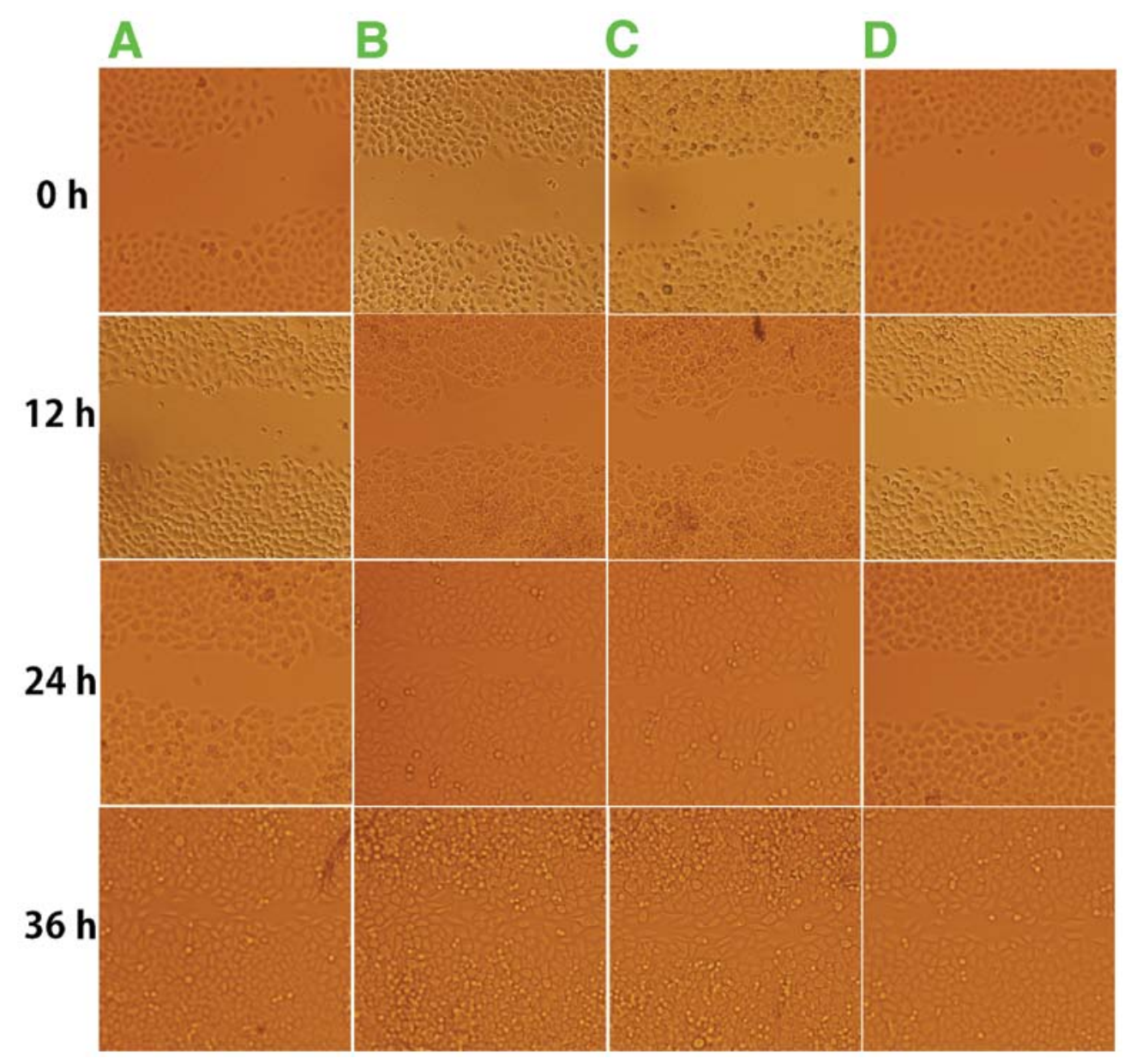

Figure 4. Wound healing capacity was monitored by microscopy after 0, 12, 24 and 36 h. Wound closure rate of F4 or F5M2 cells transfected with miR-34a mimics was significantly lower than that of F5M2 cells untreated or treated with negative controls (NC). (A) F4; (B) F5M2 untransfected; (C) F5M2 transfected with NC; (D) F5M2 transfected with miR-34a mimics.

curve was then depicted. The results demonstrated that the F5M2 cells transfected with the miR-34a mimics exhibited a similar tendency in proliferation capacity between the groups. This demonstrates that the exogenous miR-34a expression had little effect on the proliferation of osteosarcoma cells (Fig. 5C).

\section{Discussion}

It has previously been reported that several miRNAs are metastatic suppressors, including miR-126, miR-335, let-7c, miR-100, miR-218, miR-375, miR-125, miR-198 and miR-142. The reduced expression of miR-126 and miR-335 has been 

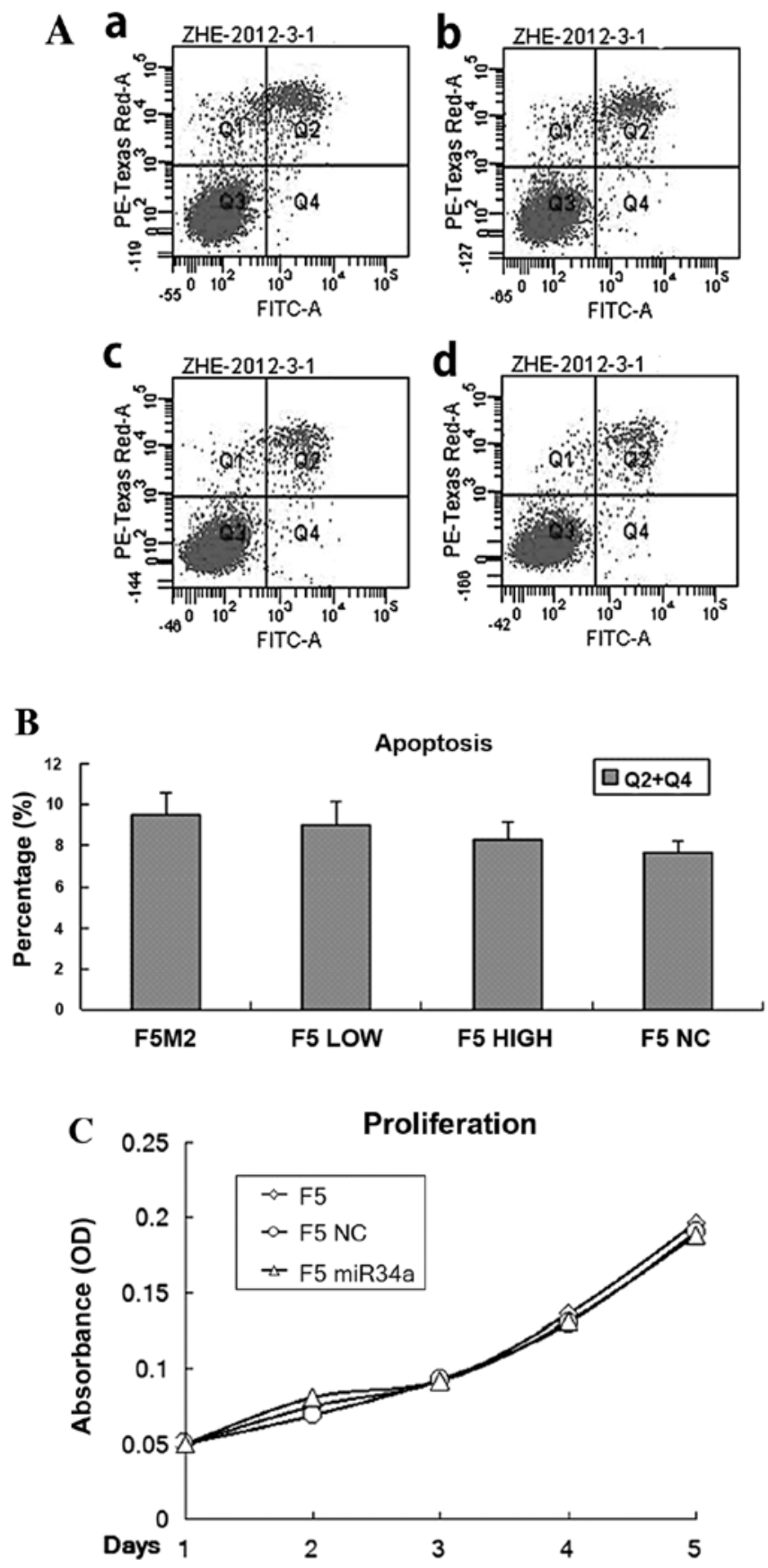

Figure 5. (A and B) There was no statistical difference in the total apoptotic ratio between F5M2 and F4 cells as well as between F5M2 cells transfected and untransfected with miR-34a. Quadrant $2(\mathrm{Q} 2)$ represents the percentage of viable apoptotic cells. Quadrant 4 (Q4) represents the percentage of nonviable apoptotic cells. Total apoptotic percentage was equal to Q2 and Q4. (a) F5M2 untransfected; (b) F5M2 transfected with low miR-34a; (c) F5M2 transfected with high miR-34a; (d) F5M2 transfected with NC. (C) The results demonstrated that F5M2 cells transfected with miR-34a mimics exhibited a similar proliferation capacity to the F5M2 cells untreated or transfected with the negative control $(\mathrm{NC})$.

found in breast cancer characterized by poor metastatic-free survival (26), while the expression of miR-let7c, miR-100 and miR-218 has been shown to be significantly decreased in metastatic prostate cancer compared with localized prostate cancer (27). The ectopic expression of miR-375 induces changes in cell morphology, and inhibits melanoma cell invasion and wound healing, strongly suggesting the functional role of miR-375 in cytoskeletal architecture and migration (28). Moreover, the enforced ectopic expression of miR-125 has been shown to impair cell migration and invasion in a breast cancer cell line, and the reduction of miR-125 expression enhances the migration ability of the cells (29). miR-198 has been shown to be downregulated in HCC and the forced ectopic expression of miR-198 represses HCC cell migration and invasion in a c-METdependent manner (30). Another study reported that miR-142 expression was downregulated in HCC cells and that the overexpression of miR-142 inhibited cell migration and invasion, while the blocking of miR-142 increased cell migration and invasion. It has also been demonstrated that miR-142 inhibits HCC cell migration and invasion by targeting RAC1 (31).

The functional analysis of miR-34a has recently been reported in mouse HCC cell lines and in human prostate cancer progenitor cells. Liu et al (21) presented miR-34a as a potent metastasis inhibitor of prostate cancer and reported that the upregulation of miR-34a inhibited the migration of cancer cells and extended animal survival. They demonstrated that miR-34a induced the dysregulation of genes related to migration and invasion by directly repressing CD44. Guo et al demonstrated that the ectopic upregulation of miR-34a inhibited the lymphatic metastatic potential of Hca-F cells (highly metastatic mouse hepatoma cells); however, this was shown to be mediated via the downregulation of cyclin D1 and CDK6 in Hepa1-6 cells (20). Some targets of miR-34a have been recently identified, including CDK4/6, CCND1, CCNE2, E2F3, Bcl-2, MYCN, c-Met, SIRT1, Notch1 and Jagged1 (14,32-35). However, the role of miR-34a in tumor metastasis remains to be fully elucidated. Taken together, these data indicate that miR-34a is likely to have a number of targets through which it regulates the biological functions of cancer cells, including metastasis.

In the results of previous biochemical studies, it has been reported that the miRNA repression of mRNA expression is dependent on specific cellular conditions (36). Studies have demonstrated that the expression profiling of miR-34a is tissuespecific, and that it may have divergent functions depending on the tumor tissue or cell type. To identify the potential role of miR-34a in osteosarcoma metastasis, we carried out this study to compare the miR-34a expression level in F5M2 cells with that in F4 cells, which are sublines of the osteosarcoma cell line, SOSP-9607, with high and low metastatic potential, respectively (18). We used a variety of methods to analyze the inhibitory role of miR-34a in the migration and invasion of F5M2 cells. Real-time PCR demonstrated that miR-34a expression in F5M2 cells was lower than that in F4 cells. After transfection with miR-34a mimics, the results indicated that the overexpression of miR-34a mainly inhibited the migration and invasion of the transfected F5M2 cells, compared with the F5M2 cells untreated or transfected with the negative controls. Therefore, it is reasonable to conclude that miR-34a may act as a tumor suppressor in osteosarcoma.

The identification of cancer-specific miRNAs and their targets is pivotal for understanding their role in tumorigenesis and metastasis, and may be important for the discovery of novel therapeutic targets. To identify the suppressive mechanism of miR-34a in osteosarcoma cell metastasis, we employed miRNA target prediction programs (TargetScan and PicTa'r) to match 
the direct targets of miR-34a (23). Markedly, the programs predicted that CD44 was one of the targets of miR-34a. CD44 which contained the corresponding binding site of miR-34a in its 3'UTR, was regulated by miR-34a. A previous study demonstrated that miR-34a inhibited prostate cancer stem cells and metastasis by directly repressing CD44 (21). The results of the present study showed that the CD44 level inversely correlated with the miR-34a level. The results of luciferase reporter assay demonstrated that CD44 was a direct target of miR-34a in F5M2 cells. Of note, this study also demonstrated that CD44 levels positively correlated with osteosarcoma cell metastasis and invasion. Therefore, it is reasonable to conclude that alterations in miR-34a expression may regulate cell migration and invasion by targeting CD44.

CD44 (also known as homing cellular adhesion molecule, Hermes antigen and PGP-1) is encoded by a single, highly conserved gene with a length of $50-60 \mathrm{~kb}$ and is composed of 20 exons. Hhuman CD44 is an 85-90 kDa transmembrane glycoprotein receptor belonging to the family of cell adhesion proteins and involved in cell-extracellular matrix interactions as well as in cell-cell interactions. CD44 participates in many cellular processes including the regulation of cell survival, migration and adhesion through the binding of its major ligand, hyaluronic acid (37-40). Recently, it has been reported that the aberrant overexpression of CD44 correlates with the metastatic potential of several malignant tumors, such as prostate cancer, breast tumors and chondrosarcoma $(21,41,42)$.

CD44 is a molecular marker in a variety of tumors. Chen et al reported that $\mathrm{CD} 133^{+} \mathrm{CD} 44^{+}$stem-like cancer cells were highly enriched in HCT116 colon cancer cells and that metastatic colon cancer cells resided exclusively in a subpopulation of cells with a high CD44 expression (43). Zhang et al strongly suggested that the $\mathrm{CD} 44^{+}$subpopulation of human gastric cancer cell lines, AGS, is gastric cancer stem cells (44). It has been reported that CD44 is involved in cancer cell adhesion, motility and invasion through the interaction with hyaluronic acid and is necessary for the metastasis and recurrence of breast cancer cells $(45,46)$. The same study also concluded that antibodies against different epitopes on CD44 mediated distinct functional effects on breast cancer cells. Heyse et al suggested that the overexpression of CD44s correlated significantly with the metastatic potential of human chondrosarcoma cells and there was a significant association beetween high CD44 expression and poor survival in patients with chondrosarcoma (42). They also reported that the overexpression of CD44 was necessary for the metastasis of osteosarcoma and that the high expression of CD44 was also associated with the low survival rate of patients with osteosarcoma (47).

The overexpression of CD44 may be considered a prognostic parameter of osteosarcoma and a metastatic parameter of chondrosarcoma $(42,47)$, but whether miRNAs regulate CD44 and osteosarcoma metastasis remains unclear. In this study, through expression analysis, we showed that miR-34a was underexpressed in highly metastatic F5M2 cells originating from the osteosarcoma cell line, SOSP-9607.

To verify the inhibitory effect of miR-34a on CD44 expression, we conducted a trial to transfect miR-34a into F5M2 cells, which expressed higher levels of CD44 protein and had a higher pulmonary metastatic potential than the paired F4 cells (18). After transfection with miR-34a mimics, CD44 expression in
F5M2 cells was almost abolished, as shown by western blot analysis and ICC. On the contrary, F5M2 cells transfected with the negative controls or miR-34a inhibitors showed only a slight change in CD44 expression. Our study also showed that the enforced ectopic expression of miR-34a in F5M2 cells significantly inhibited cell motility and invasion. We also demonstrated that miR-34a had little effect on cell apoptosis and proliferation. Taken together, these data suggest that miR-34a expression is inversely related to the metastasis of osteosarcoma cells. CD44 expression positively correlated with the metastasis and invasion of osteosarcoma cells. Therefore, it can be concluded that the enforced ectopic expression of miR-34a in F5M2 cells might act as a significant inhibitory factor in the progression of osteosarcoma cells by downregulating the expression of CD44. Of note, CD44 was identified and validated as a direct and functional target of miR-34a in inhibiting osteosarcoma cell regeneration and metastasis.

Many human cancers contain cancer stem cells or tumor progenitor cells, which have the ability of self-renewal, partial recreation of the cellular heterogeneity of the parental cells, and possess an enhanced tumor-initiating capacity, and are thus more resistant than most general cancer cells to conventional anticancer treatment. Due to these properties, cancer stem cells or tumor progenitor cells have been linked to tumor progression and metastasis (48). Chen et al demonstrated with clonal analysis that a lineage of self-renewing tumor-initiating cell types existed in glioblastoma (49). Either individually or in combination with other markers, the adhesion molecule, CD44, has identified cancer stem cells in many tumors, including cancers of the breast, pancreas, head and neck, colon, small intestine, live, stomach, bladder and ovaries.

The exact mechanisms by which miR-34a inhibits osteosarcoma cell metastasis by repressing CD44 still remain largely ambiguous; however, the enforced ectopic expression of miR-34a inhibited the migration and invasion of the F5M2 cell subline. Considering the widespread expression of CD44 in cancer stem cells and the functional involvement of CD44 in mediating cancer stem cell migration and the metastasis of many cancers $(50,51)$, the newly identified miR-34a suppression of CD44 reveals a key role for miRNA-based gene regulation. The emerging tumor-suppressive role of miR-34a in prostate and lung tumors $(21,52)$, establishes a strong rationale for developing miR-34a as a novel therapy targeting osteosarcoma cells.

During the course of our study, other reports on miRNAs were published, which identified miR-219, miR-20a, miR-223, miR-222, miR-195 tumor-associated miRNAs. miR-219 has been shown to exert tumor-suppressive effects in hepatic carcinogenesis through the negative regulation of GPC3 expression in vitro (53). miR-20a promotes the migration and invasion of cervical cancer cells through the direct upregulation of TNKS2, which induces the colony formation, migration and invasion of cervical cancer cells (54). The overexpression of miR-223 significantly inhibits cell proliferation by regulating FOXO1 expression (55). These studies further suggest that miRNAs are involved in tumor metastasis and progression.

The findings of this study illustrate that, by downregulating CD44 expression, miR-34a plays an inhibitory role in the migration and invasion of osteosarcoma cells. To our knowledge, this is the first study to demonstrate that miR-34a 
regulates the metastasis and progression of osteosarcoma cells, by targeting the expression of CD44 in F5M2 cells in vitro. The data presented in our study may provide an novel avenue for further analysis with an aim to develop a novel potential therapeutic target for the treatment of highly metastatic osteosarcoma in vivo. Further study would be required to fully elucidate the exact roles of miR-34a and CD44 in osteosarcoma in vitro and in vivo.

\section{Acknowledgements}

We would like to thank Yanhua Wen, Chen Xiang, Yunyan Liu, Dianzhong Zhang and Hong Zhao for their excellent technical assistance and helpful discussions.

\section{References}

1. Bentzen SM, Poulsen HS, Kaae S, Jensen OM, Johansen H, Mouridsen HT, Daugaard S and Arnoldi C: Prognostic factors in osteosarcomas, a regression analysis. Cancer 62: 194-202, 1988.

2. Davis AM, Bell RS and Goodwin PJ: Prognostic factors in osteosarcoma: a critical review. J Clin Oncol 12: 423-431, 1994.

3. Mankin HJ, Hornicek FJ, Rosenberg AE, Harmon DC and Gebhardt MC: Survival data for 648 patients with osteosarcoma treated at one institution. Clin Orthop Relat Res 429: 286-291, 2004.

4. Filipowicz W, Bhattacharyya SN and Sonenberg N: Mechanisms of post-transcriptional regulation by microRNAs: are the answers in sight? Nat Rev Genet 9: 102-114, 2008.

5. Medina PP and Slack FJ: microRNAs and cancer: an overview. Cell Cycle 7: 2485-2492, 2008.

6. Pillai RS, Bhattacharyya SN and Filipowiczm W: Repression of protein synthesis by miRNAs: how many mechanisms? Trends Cell Biol 17: 118-126, 2007.

7. Bartel DP: MicroRNAs: genomics, biogenesis, mechanism, and function. Cell 116: 281-297, 2004.

8. Zhang B, Pan X, Cobb GP and Anderson TA: microRNAs as oncogenes and tumor suppressors. Dev Biol 302: 1-12, 2007.

9. Bartel DP: MicroRNAs: target recognition and regulatory functions. Cell 136: 215-233, 2009.

10. Caldas C and Brenton JD: Sizing up miRNAs as cancer genes. Nat Med 11: 712-714, 2005.

11. He L, He X, Lim LP, de Stanchina E, Xuan Z, et al: A microRNA component of the p53 tumour suppressor network. Nature 447: $1130-1134,2007$.

12. Raver-Shapira N, Marciano E, Meiri E, Spector Y, Rosenfeld N, et al: Transcriptional activation of miR-34a contributes to p53-mediated apoptosis. Mol Cell 26: 731-743, 2007.

13. Welch C, Chen Y and Stallings RL: MicroRNA-34a functions as a potential tumor suppressor by inducing apoptosis in neuroblastoma cells. Oncogene 26: 5017-5022, 2007.

14. Lewis BP, Burge CB and Bartel DP: Conserved seed pairing, often flanked by adenosines, indicates that thousands of human genes are microRNA targets. Cell 120: 15-20, 2005.

15. Sun F, Fu H, Liu Q, Tie Y, Zhu J, et al: Downregulation of CCND1 and CDK6 by miR-34a induces cell cycle arrest. FEBS Lett 582: 1564-1568, 2005.

16. Wei JS, Song YK, Durinck S, Chen QR, Cheuk AT, et al: The MYCN oncogene is a direct target of miR-34a. Oncogene 27: 5204-5213, 2008.

17. Yamakuchi M, Ferlito M and Lowenstein CJ: miR-34a repression of SIRT1 regulates apoptosis. Proc Natl Acad Sci USA 105: 13421-13426, 2008.

18. Chen X, Yang TT, Wang W, Sun HH, Ma BA, Li CX, Ma Q, $\mathrm{Yu} \mathrm{Z}$ and Fan QY: Establishment and characterization of human osteosarcoma cell lines with different pulmonary metastatic potentials. Cytotechnology 61: 37-44, 2009.

19. He C, Xiong J, Xu X, Lu W, Liu L, Xiao D and Wang D: Functional elucidation of MiR-34 in osteosarcoma cells and primary tumor samples. Biochem Biophys Res Commun 388: 35-40, 2009.

20. Guo Y, Li S, Qu J, Wang S, Dang Y, et al: MiR-34a inhibits lymphatic metastasis potential of mouse hepatoma cells. Mol Cell Biochem 354: 275-282, 2011.
21. Liu C, Kelnar K, Liu B, Chen X, Calhoun-Davis T, et al: The microRNA miR-34a inhibits prostate cancer stem cells and metastasis by directly repressing CD44. Nat Med 17: 211-215, 2011.

22. Lewis BP, Shih IH, Jones-Rhoades MW, Bartel DP and Burge CB: Prediction of mammalian microRNA targets. Cell 115: 787-798, 2003.

23. Krek A, Grün D, Poy MN, Wolf R, Rosenberg L, et al: Combinatorial microRNA target predictions. Nat Genet 37: 495-500, 2005

24. John B, Enright AJ, Aravin A, Tuschl T, Sander C and Marks DS: Human MicroRNA targets. PLoS Biol 2: e264, 2004.

25. Coltella N, Manara MC, Cerisano V, Trusolino L, et al: Role of the MET/HGF receptor in proliferation and invasive behavior of osteosarcoma. FASEB J 17: 1162-1164, 2003.

26. Tavazoie SF, Alarcón C, Oskarsson T, Padua D, Wang Q, et al: Endogenous human microRNAs that suppress breast cancer metastasis. Nature 451: 147-152, 2008.

27. Leite KR, Sousa-Canavez JM, Reis ST, Tomiyama AH, CamaraLopes LH, Sañudo A, Antunes AA and Srougi M: Change in expression of miR-let7c, miR-100, and miR-218 from high grade localized prostate cancer to metastasis. Urol Oncol 29: 265-269, 2009.

28. Mazar J, DeBlasio D, Govindarajan SS, Zhang S and Perera RJ: Epigenetic regulation of microRNA-375 and its role in melanoma development in humans. FEBS Lett 585: 2467-2476, 2011.

29. Kumar MS, Lu J, Mercer KL, Golub TR and Jacks T: Impaired microRNA processing enhances cellular transformation and tumorigenesis. Nat Genet 39: 673-677, 2007.

30. Tan S, Li R, Ding K, Lobie PE and Zhu T: miR-198 inhibits migration and invasion of hepatocellular carcinoma cells by targeting the HGF/c-MET pathway. FEBS Lett 585: 2229-2234, 2011.

31. Wu L, Cai C, Wang X,Liu M,Li X and Tang H: MicroRNA-142-3p, a new regulator of $\mathrm{RAC1}$, suppresses the migration and invasion of hepatocellular carcinoma cells. FEBS Lett 585: 1322-1330, 2011.

32. Cozzolino AM, Pedace L, Castori M, De Simone P, Preziosi N, et al: Analysis of the miR-34a locus in 62 patients with familial cutaneous melanoma negative for CDKN2A/CDK4 screening. Fam Cancer 11: 201-208, 2012.

33. Yan D, Zhou X, Chen X, Hu D, Dong X, et al: MicroRNA-34a Inhibits uveal melanoma cell proliferation and migration through downregulation of c-Met. Invest Ophthalmol Vis Sci 50: $1559-1565,2009$.

34. Zhao T, Li J and Chen AF: MicroRNA-34a induces endothelial progenitor cell senescence and impedes its angiogenesis via suppressing silent information regulator 1 . Am J Physiol Endocrinol Metab 299: E110-E116, 2010.

35. Pang RT, Leung CO, Ye TM, Liu W, Chiu PC, et al: MicroRNA-34a suppresses invasion through downregulation of Notch1 and Jagged1 in cervical carcinoma and choriocarcinoma cells. Carcinogenesis 31: 1037-1044, 2010.

36. Doench JG and Sharp PA: Specificity of microRNA target selection in translational repression. Genes Dev 18: 504-511, 2004.

37. Aruffo A, Stamenkovic I, Melnick M, Underhill CB and Seed B: CD44 is the principal cell surface receptor for hyaluronate. Cell 61: 1303-1313, 1990.

38. Ponta H, Sherman L and Herrlich PA: CD44: from adhesion molecules to signalling regulators. Nat Rev Mol Cell Biol 4: 33-45, 2003.

39. Marhaba R and Zöller M: CD44 in cancer progression: adhesion, migration and growth regulation. J Mol Histol 35: 211-231, 2004.

40. Gotte $\mathrm{M}$ and Yip GW: Heparanase, hyaluronan, and CD44 in cancers: a breast carcinoma perspective. Cancer Res 66: 10233-10237, 2006.

41. Olsson E, Honeth G, Bendahl PO, Saal LH, Gruvberger-Saal S, Ringnér M, et al: CD44 isoforms are heterogeneously expressed in breast cancer and correlate with tumor subtypes and cancer stem cell markers. BMC Cancer 11: 418, 2011.

42. Heyse TJ, Malcherczyk D, Moll R, Timmesfeld N, Wapelhorst J, et al: CD44: survival and metastasis in chondrosarcoma. Osteoarthritis Cartilage 18: 849-856, 2010.

43. Chen KL, Pan F, Jiang H, Chen JF, Pei L, Xie FW and Liang HJ: Highly enriched CD133 ${ }^{+} \mathrm{CD} 44^{+}$stem-like cells with $\mathrm{CD} 133^{+} \mathrm{CD} 44^{\text {high }}$ metastatic subset in HCT116 colon cancer cells . Clin Exp Metastasis 28: 751-763, 2011.

44. Zhang C, Li C, He F, Cai Y and Yang H: Identification of $\mathrm{CD} 44^{+} \mathrm{CD} 24^{+}$gastric cancer stem cells. J Cancer Res Clin Oncol 137: 1679-1686, 2011. 
45. Takaishi S, Okumura T, Tu S, Wang SS, Shibata W, et al: Identification of gastric cancer stem cells using the cell surface marker CD44. Stem Cells 27: 1006-1020, 2009.

46. Afify A, Purnell P and Nguyen L: Role of CD44s and CD44v6 on human breast cancer cell adhesion, migration, and invasion. Exp Mol Pathol 86: 95-100, 2009.

47. Kim HS, Park YB, Oh JH, Jeong J, Kim CJ and Lee SH: Expression of CD44 isoforms correlates with the metastatic potential of osteosarcoma. Clin Orthop Relat Res 396: 184-190, 2002.

48. Visvader JE and Lindeman GJ: Cancer stem cells in solid tumours: accumulating evidence and unresolved questions. Nat Rev Cancer 8: 755-768, 2008.

49. Chen R, Nishimura MC, Bumbaca SM, et al: A hierarchy of self-renewing tumor-initiating cell types in glioblastoma. Cancer Cell 17: 362-375, 2010.

50. Jin L, Hope KJ, Zhai Q, Smadja-Joffe F and Dick JE: Targeting of CD44 eradicates human acute myeloid leukemic stem cells. Nat Med 12: 1167-1174, 2006.
51. Ji Q, Hao X, Zhang M, Tang W, Yang M, et al: MicroRNA miR-34 inhibits human pancreatic cancer tumor-initiating cells. PLoS One 4: e6816, 2009.

52. Wiggins JF, Ruffino L, Kelnar K, Omotola M, Patrawala L, et al: Development of a lung cancer therapeutic based on the tumor suppressor microRNA-34. Cancer Res 70: 5923-5930, 2010.

53. Huang N, Lin J, Ruan J, Su N, Qing R, Liu F, He B, Lv C, Zheng D and Luo R: MiR-219-5p inhibits hepatocellular carcinoma cell proliferation by targeting glypican-3. FEBS Lett 586: 884-891, 2012.

54. Kang HW, Wang F, Wei Q, Zhao YF, Liu M, Li X and Tang H: miR-20a promotes migration and invasion by regulating TNKS2 in human cervical cancer cells. FEBS Lett 586: 897-904, 2012

55. Wu L, Li H, Jia CY, Cheng W, Yu M, Peng M, Zhu Y, Zhao Q, Dong YW, Shao K, Wu A and Wu XZ: MicroRNA-223 regulates FOXO1 expression and cell proliferation. FEBS Lett 586: 1038-1043, 2012. 